\title{
Efficient Capture and T2 Magnetic Resonance Assay of Candida albicans with Inorganic Nanoparticles: Role of Nanoparticle Surface Charge and Fungal Cell Wall
}

Wei Tian ${ }^{1,2}$, Fan $\mathrm{Li}^{3}$, Shengming $\mathrm{Wu}^{2}$, Gen $\mathrm{Li}^{4}$, Lieying $\mathrm{Fan}^{4}, \mathrm{Xue} \mathrm{Qu}^{1,5 *}$, Xinming $\mathrm{Jia}^{3 *}$, Yilong Wang ${ }^{2 *}$

1. Key Laboratory for Ultrafine Materials of Ministry of Education, School of Materials Science and Engineering, East China University of Science and Technology, Shanghai 200237, P.R. China.E-mail: quxue@ecust.edu.cn

2. The Institute for Translational Nanomedicine, Shanghai East Hospital, the Institute for Biomedical Engineering \& Nano Science, Tongji University School of Medicine, Shanghai 200092, P. R. China. Email: yilongwang@ tongji.edu.cn.

3. Clinical Translational Research Center, Shanghai Pulmonary Hospital, Tongji University School of Medicine, Shanghai, P. R. China. Email: Jiaxm@tongji.edu.cn

4. Department of Clinical Lab, Shanghai East Hospital, Tongji University School of Medicine, Shanghai 200092, P. R. China.

5. State Key Laboratory of Molecular Engineering of Polymers, Fudan University, Shanghai, 200433, P. R. China. 


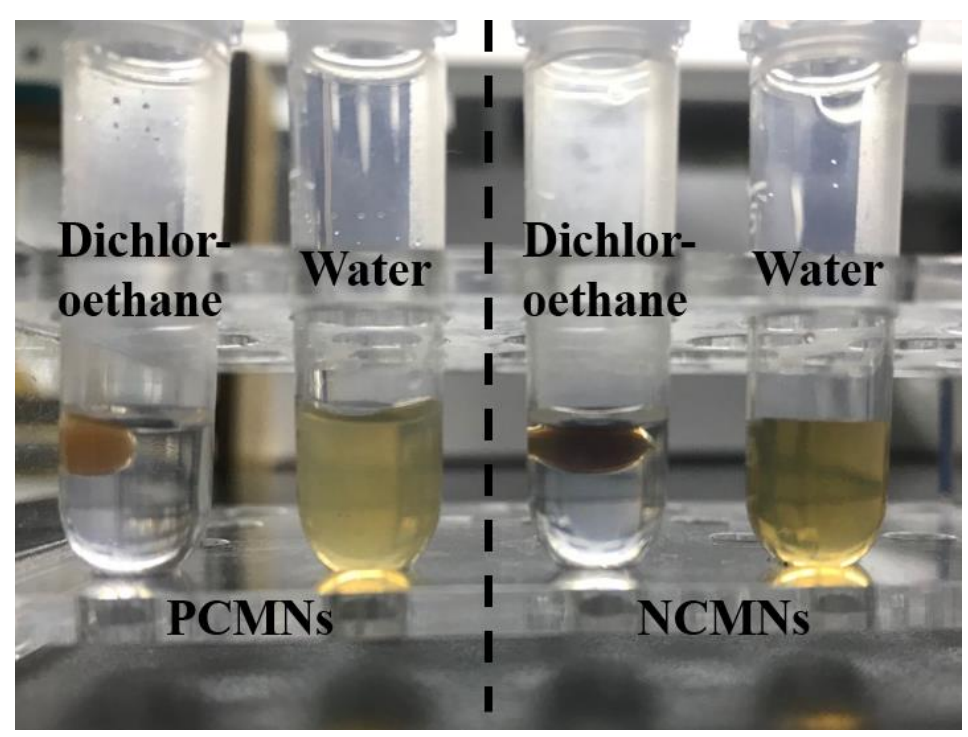

Figure S1. Photographs of positively-charged (PCMNs) and negatively-charged (NCMNs) nanoparticles dispersed in Dichloroethane and DI water respectively. 

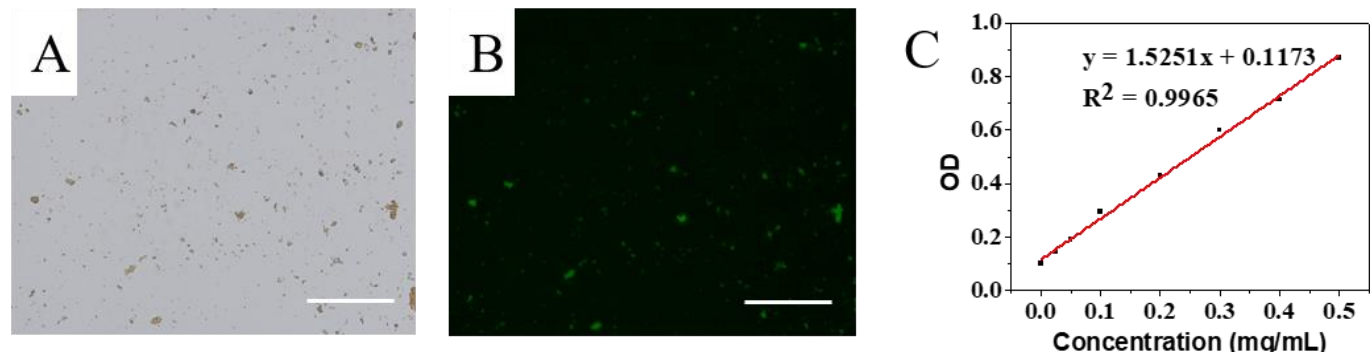

Figure S2. Fluorescent microscopy images of FITC-conA conjugated carboxylate functionalized magnetic nanoparticles: (A) fluorescence microscopy and (B) bright field images. (C) The standard curve of concentration of conA and its optical density value measure by BCA kit. 
Table S1. Ratio of capture efficiency of yeast strains by the PCMNs and NCMNs.

\begin{tabular}{llll}
\hline Concentration $(\mathrm{mg} / \mathrm{mL})$ & 150 & 100 & 50 \\
\hline Ratio $(\mathrm{PCMN} / \mathrm{NCMN})$ & 3.3 & 4.9 & 5.2 \\
\hline
\end{tabular}




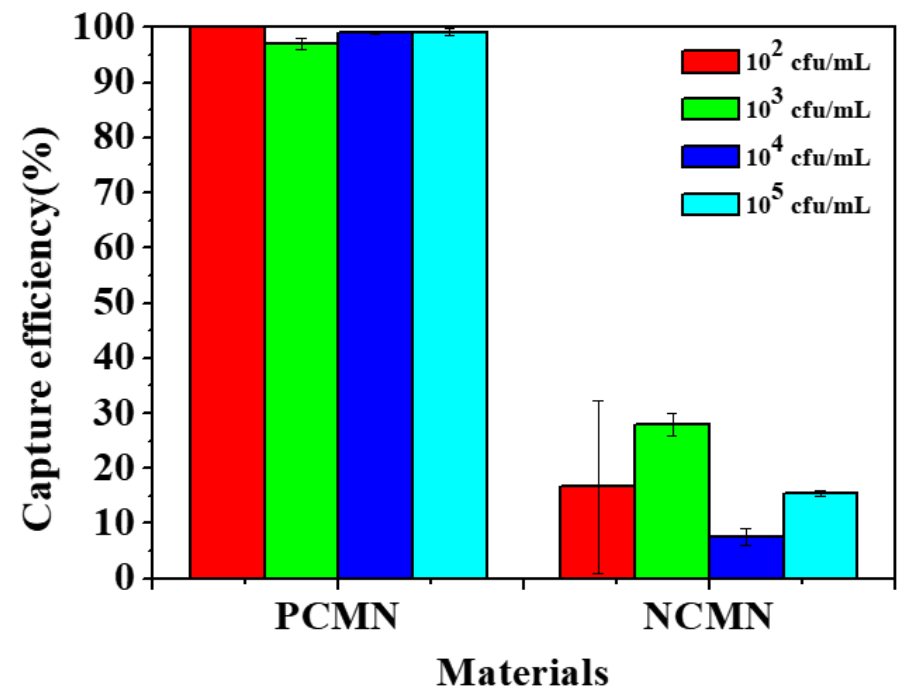

Figure S3. Comparison of the capture efficiencies of $C$. albicans at different original concentrations in the ultra-pure water by PCMNs and NCMNs. 


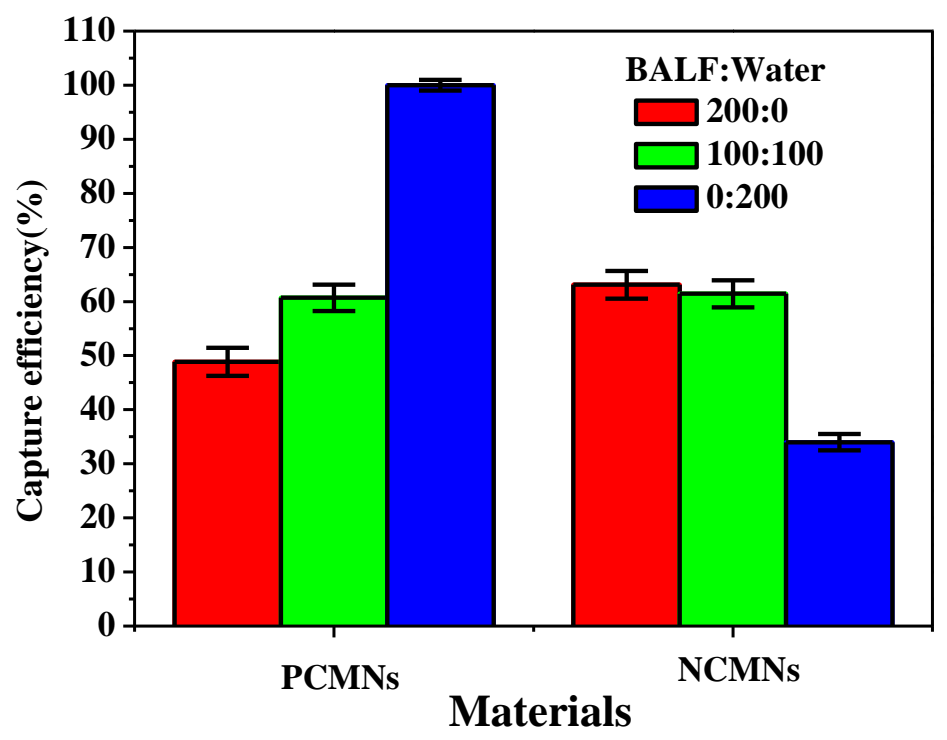

Figure S4. Comparison of capture efficiencies of the $C$. albicans spiked into the clinical sample BALF by two kinds of surface charged MNs in different medium. 


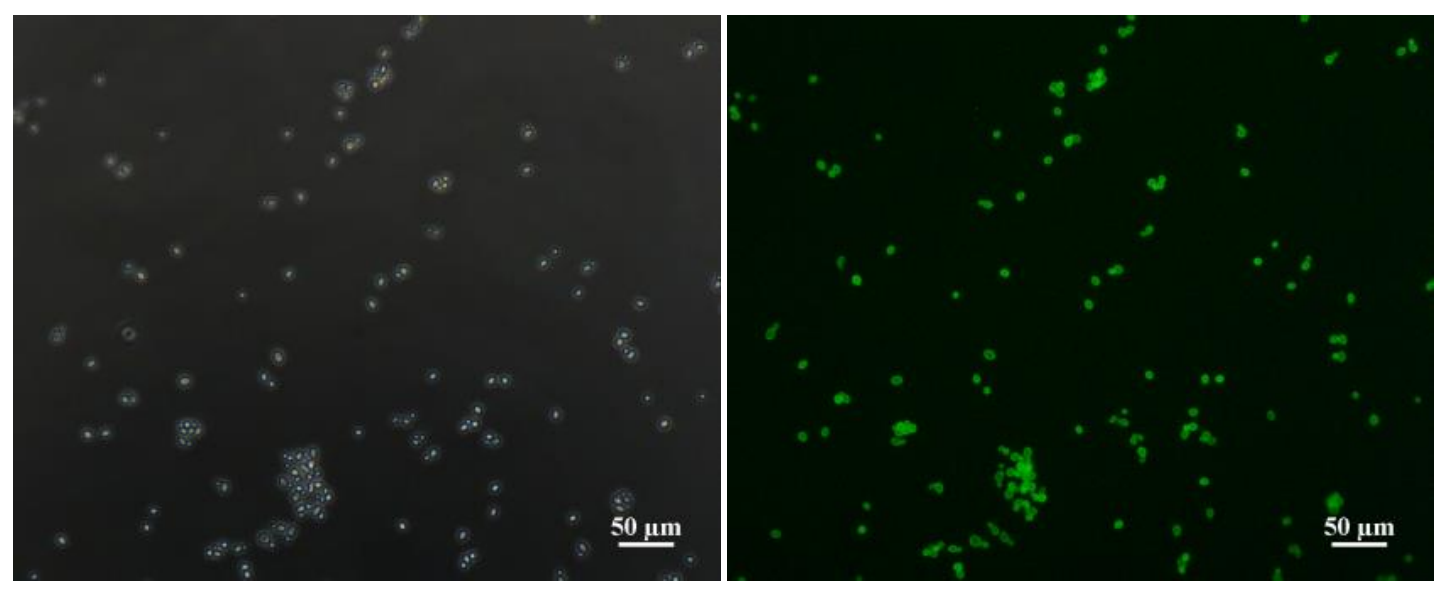

Figure S5. Bright field and fluorescence microscopy images of $C$. albicans labelled by FITC-conA. 


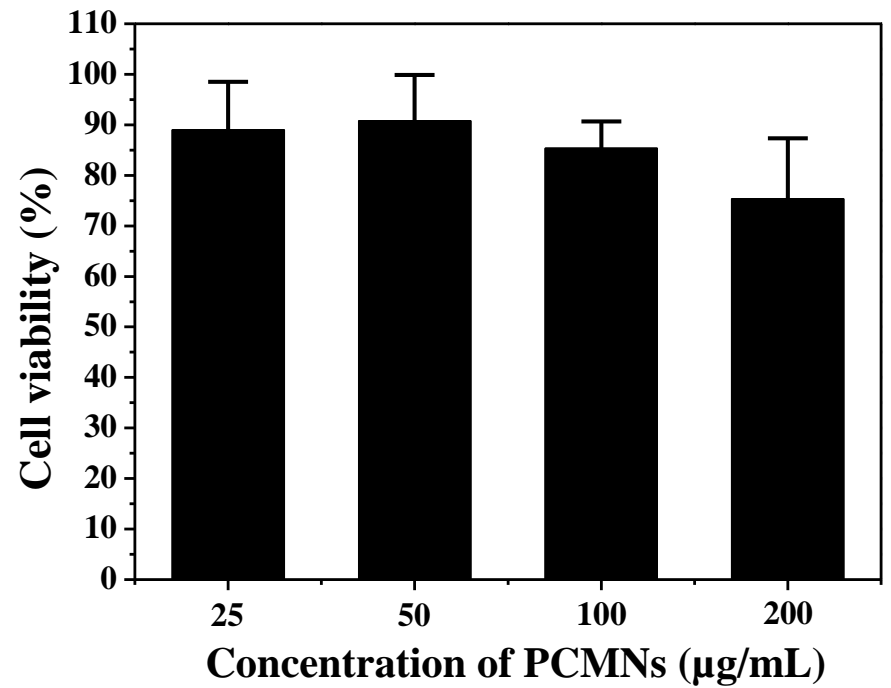

Figure S6. Cellular viability of $C$. albicans yeast treated by the positively-charged magnetic nanoparticles at different concentrations. 